\title{
Mitochondrial transplantation therapy for ischemia reperfusion injury: a systematic review of animal and human studies
}

\author{
Kei Hayashida ${ }^{1,2^{*}}$ (D), Ryosuke Takegawa ${ }^{1,2}$, Muhammad Shoaib ${ }^{1,2,3}$, Tomoaki Aoki ${ }^{1,2}$, Rishabh C. Choudhary ${ }^{1,2}$, \\ Cyrus E. Kuschner ${ }^{1,2,3}$, Mitsuaki Nishikimi 1,2, Santiago J. Miyara ${ }^{1,2}$, Daniel M. Rolston²,3, Sara Guevara4, \\ Junhwan Kim ${ }^{1,2,3}$, Koichiro Shinozaki ${ }^{1,2,3}$, Ernesto P. Molmenti ${ }^{3,4}$ and Lance B. Becker ${ }^{1,2,3}$
}

\begin{abstract}
Background: Mitochondria are essential organelles that provide energy for cellular functions, participate in cellular signaling and growth, and facilitate cell death. Based on their multifactorial roles, mitochondria are also critical in the progression of critical illnesses. Transplantation of mitochondria has been reported as a potential promising approach to treat critical illnesses, particularly ischemia reperfusion injury (IRI). However, a systematic review of the relevant literature has not been conducted to date. Here, we systematically reviewed the animal and human studies relevant to IRI to summarize the evidence for mitochondrial transplantation.
\end{abstract}

Methods: We searched MEDLINE, the Cochrane library, and Embase and performed a systematic review of mitochondrial transplantation for IRI in both preclinical and clinical studies. We developed a search strategy using a combination of keywords and Medical Subject Heading/Emtree terms. Studies including cell-mediated transfer of mitochondria as a transfer method were excluded. Data were extracted to a tailored template, and data synthesis was descriptive because the data were not suitable for meta-analysis.

Results: Overall, we identified 20 animal studies and two human studies. Among animal studies, 14 (70\%) studies focused on either brain or heart IRI. Both autograft and allograft mitochondrial transplantation were used in 17 (85\%) animal studies. The designs of the animal studies were heterogeneous in terms of the route of administration, timing of transplantation, and dosage used. Twelve (60\%) studies were performed in a blinded manner. All animal studies reported that mitochondrial transplantation markedly mitigated IRI in the target tissues, but there was variation in biological biomarkers and pathological changes. The human studies were conducted with a single-arm, unblinded design, in which autologous mitochondrial transplantation was applied to pediatric patients who required extracorporeal membrane oxygenation (ECMO) for IRI-associated myocardial dysfunction after cardiac surgery.

Conclusion: The evidence gathered from our systematic review supports the potential beneficial effects of mitochondrial transplantation after IRI, but its clinical translation remains limited. Further investigations are thus required to explore the mechanisms of action and patient outcomes in critical settings after mitochondrial transplantation. Systematic review registration The study was registered at UMIN under the registration number UMIN000043347.

Keywords: Systematic review, Mitochondria, Transplantation, Ischemia reperfusion injury, Translation science

*Correspondence: khayashida@northwell.edu

${ }^{1}$ The Feinstein Institutes for Medical Research, Northwell Health System,

350 Community Drive, Manhasset, NY, USA

Full list of author information is available at the end of the article

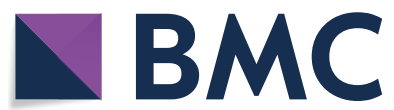

(c) The Author(s) 2021. This article is licensed under a Creative Commons Attribution 4.0 International License, which permits use, sharing, adaptation, distribution and reproduction in any medium or format, as long as you give appropriate credit to the original author(s) and the source, provide a link to the Creative Commons licence, and indicate if changes were made. The images or other third party material in this article are included in the article's Creative Commons licence, unless indicated otherwise in a credit line to the material. If material is not included in the article's Creative Commons licence and your intended use is not permitted by statutory regulation or exceeds the permitted use, you will need to obtain permission directly from the copyright holder. To view a copy of this licence, visit http://creativeco mmons.org/licenses/by/4.0/. The Creative Commons Public Domain Dedication waiver (http://creativecommons.org/publicdomain/ zero/1.0/) applies to the data made available in this article, unless otherwise stated in a credit line to the data. 


\section{Background}

Mitochondrial transplantation is a therapeutic approach that involves injection of healthy mitochondria into damaged organs. Recent evidence has shown that the physiological properties of healthy mitochondria provide the possibility of replacing damaged mitochondria $[1,2]$, suggesting that replacement of damaged mitochondria with healthy mitochondria may protect cells against further injury [3]. Furthermore, mitochondria might also be actively released into the extracellular space and potentially be transferred from cell to cell in the central nervous system [4]. This heightened interest in mitochondrial therapy calls for a deeper understanding of the mechanisms underlying mitochondrial transfer, uptake, and cellular protection [5].

Alterations in mitochondrial properties and functions play a role in modulating the development of ischemia-reperfusion injury (IRI) in a variety of critical illnesses such as sudden cardiac arrest, stroke, myocardial infarction, and major organ transplants [6-12]. IRI alters the mitochondrial electron transport chain, produces an excess of reactive oxygen species, affects mitochondrial calcium transport and calcium concentration, drives apoptotic pathways, changes mitochondrial fission/fusion dynamics and their shape, and activates mitophagy pathways [13-21]. These pathophysiological alterations lead to neurocognitive sequelae, multiple organ failure, skeletal muscle dysfunction, dysregulation of immune cell responses, and release of mitochondrial DNA, which is now recognized as a trigger for systemic inflammatory responses and a prognostic marker in critical illness [20]. Therefore, the development of "mitochondria-targeting" approaches is an unmet medical need to improve the prognosis of critically ill patients.

Since the first study demonstrating mitochondrial transplantation as a promising therapy in 2009 [3], the effects of mitochondrial transplantation have been reported in different animal models of critical illness $[4,22-25]$. However, the current evidence for the beneficial effects of mitochondrial transplantation in both preclinical and clinical settings has not been summarized systematically. Considering that systematic reviews (SR) of animal studies have been recently highlighted for improving the transparency and accessibility of the available evidence [26, 27], SRs of both preclinical and clinical efficacy studies are extremely valuable for promoting clinical translation [26]. Therefore, we conducted a systematic review of the relevant animal and human studies to summarize the current evidence of mitochondrial transplantation as a novel therapeutic strategy for IRI under critical conditions.

\section{Methods}

This study complied with the recommendations for conducting and reporting systematic reviews as set forth by the Preferred Reporting Items for Systematic Reviews and Meta-Analyses (PRISMA) statement [28]. We developed a protocol before conducting the analysis and registered it in the UMIN-CTR (registration no. UMIN000043347). The PRISMA checklist is shown in Additional file 1.

\section{Search strategy}

A comprehensive search of three major databases of biomedical publications was performed on February 17, 2021 as follows: MEDLINE (source: PubMed, 1966 to February 2021), Cochrane Central Register of Controlled Trials (through February 2021), and Embase (1974 to February 2021). We developed a search strategy using a combination of the following keywords and Medical Subject Heading (MeSH)/ Emtree terms: "(("Mitochondria"[MeSH Terms]) OR ("Mitochondrial"[tiab])) AND ("transplantation"[MeSH Terms] OR "Mitochondrial transplantation"[tiab])) AND (("Craniocerebral Trauma"[MeSH Terms]) OR (("Reperfusion Injury"[MeSH Terms]) OR "Ischemia"[MeSH Terms] OR "Ischemi*"[tiab] OR "Ischemiareperfusion"[tiab] OR "Heart arrest"[MeSH Terms]))." We did not apply any language restrictions to the electronic search. Citations were stored, and duplicates were removed using EndNote software (Thomson Reuters, Toronto, Ontario, Canada) and Rayyan software [29].

\section{Study selection and inclusion criteria}

Two independent reviewers ( $\mathrm{KH}$ and $\mathrm{RT}$ ) screened the abstracts and titles of the studies, and subsequently reviewed the full-text articles for inclusion using Rayyan software [29].

We included studies with the following characteristics:

1. Study type: investigations in both laboratory and clinical settings

2. Target models/diseases: critical illness related to IRI, including myocardial infarction, organ transplantation, cardiac arrest, and acute brain injury. Studies on chronic central nervous system diseases, and on ex vivo animal models were excluded.

3. Intervention: intraarterial or intravenous injection, of isolated mitochondria, or their direct injection into the region at risk. Studies that used cell-mediated transfer of mitochondria as a transfer method and demonstrated transfer under cell culture conditions were excluded. 
4. Control: Placebo or no intervention (without mitochondrial transplantation).

5. Outcome: biological or physiological measures of the IRI.

\section{Data extraction}

Two independent reviewers ( $\mathrm{KH}$ and $\mathrm{RT}$ ) extracted the data using a standardized data extraction form, with disagreements resolved by discussion and consensus. To ensure that no duplicate data were analyzed, the reviewers assessed the studies carefully when multiple publications by the same investigator were found. We identified the following information for each study: primary author's name, publication year, experimental model or patient population, randomization, blinded assessment, types of mitochondrial transplantation, and the main outcome. As the type and range of studies varied widely and were mostly animal models, we did not undertake formal quality assessment using an existing tool. The risk of bias across studies was not assessed. Instead, we qualitatively documented the potentially important limitations for each study. Evidence synthesis was descriptive because the data were not suitable for meta-analysis.

\section{Results}

\section{Literature search}

Figure 1 shows the PRISMA flowchart of study selection in the systematic review. The search identified 1430 records. After removing 326 duplicate articles, 1104 articles were selected for title and abstract screening. Following screening, 33 articles were selected for full-text analysis. After full-text review, 11 articles did not meet the inclusion criteria and 22 articles were included in the review; of these, 20 were animal studies and 2 were human studies.

\section{Animal studies}

A summary of the animal studies eligible for this review is presented in Table 1.

\section{Brain IRI model}

Five experimental models targeting brain injury were generated in mice [25] and rats [30-33], including four focal brain ischemia models (middle cerebral artery occlusion [MCAO]) [25, 31-33] and one traumatic brain injury (TBI) model [30]. The types of transplantation used were autologous [32], allograft [25, 30], and xenograft $[31,33]$. The types of administration were direct or near-direct injection [30-33] and intravenous [25].

Four animal studies demonstrated the benefits of mitochondrial transplantation in the setting of stroke. Using a 60 min focal carotid occlusion model in C57BL6 male mice, Nakamura et al. [25] assessed the impact of transfusing mitochondrial-enriched fractions obtained from snap-frozen placenta on the infarct size. Treatment with placental mitochondria significantly decreased the infarct size assessed using 2,3,5-triphenyltetrazolium chloride (TTC) staining at $72 \mathrm{~h}$ after reperfusion; further, fluorescent Mitotracker Deep Red imaging demonstrated diffuse mitochondrial uptake in the brain, lung liver, and kidney tissues at $2 \mathrm{~h}$ after treatment [25]. Excess reactive oxygen species and oxidative stress are major contributors to brain damage following acute ischemic stroke. Pourmohammadi-Bejarpasi et al. [31] reported the intracerebroventricular transplantation of isolated mitochondria from human umbilical cord-derived mesenchymal stem cells (hUC-MSCs) in a rat model of MCAO. MCAO occlusion in rats for $70 \mathrm{~min}$ followed by reperfusion and mitochondrial transplantation demonstrated normal brain cytoarchitecture and neurons with a decreased number of pyknotic cells [31]. Brain injury after acute stroke can result in astrocyte hypertrophy, and the subsequent release of glial fibrillary acidic protein (GFAP) can lead to scar formation, thus limiting neuronal recovery. Zhang et al. [32] demonstrated the neuroprotective effects of autologous mitochondrial transplantation obtained from the pectoralis major to the lateral ventricles after $90 \mathrm{~min}$ of MCAO. Twenty-four hours after $\mathrm{MCAO}$, the number of viable mitochondria observed in the cerebrospinal fluid were increased, which resulted in improved neurological outcomes, suggesting the potential application of mitochondrial transplantation after stroke. Infusion of isolated mitochondria in the lateral ventricles post-stroke resulted in increased mitochondrial accumulation in the penumbra with an increase in overall ATP content and in the expression of complex IV. Post-MCAO transplantation of mitochondria also reduced apoptosis, attenuated astrogliosis with increased neurogenesis, and decreased the brain infarct volume [32]. Huang et al. [33] used a male rat MCAO cerebral stroke model to compare the effects of direct intracerebral injection $v s$. intra-femoral transfusion on functional status and infarct size. Intracerebral and intra-femoral transplantation improved the functional rotarod and grip strength performance measured up to 1-month posttransplantation, significantly decreased the lesion size as estimated using Terminal deoxynucleotidyl transferase dUTP nick end labeling (TUNEL) assay, and showed diffuse distribution of the grafted mitochondria in neurons, astrocytes, and microglia. Mitochondria treated with antimycin A and oligomycin failed to provide equivalent protection against oxygen glucose deprivation (OGD)induced stress, suggesting that transplantation benefits require intact mitochondrial function [33]. 


\section{PRISMA Flow Diagram}

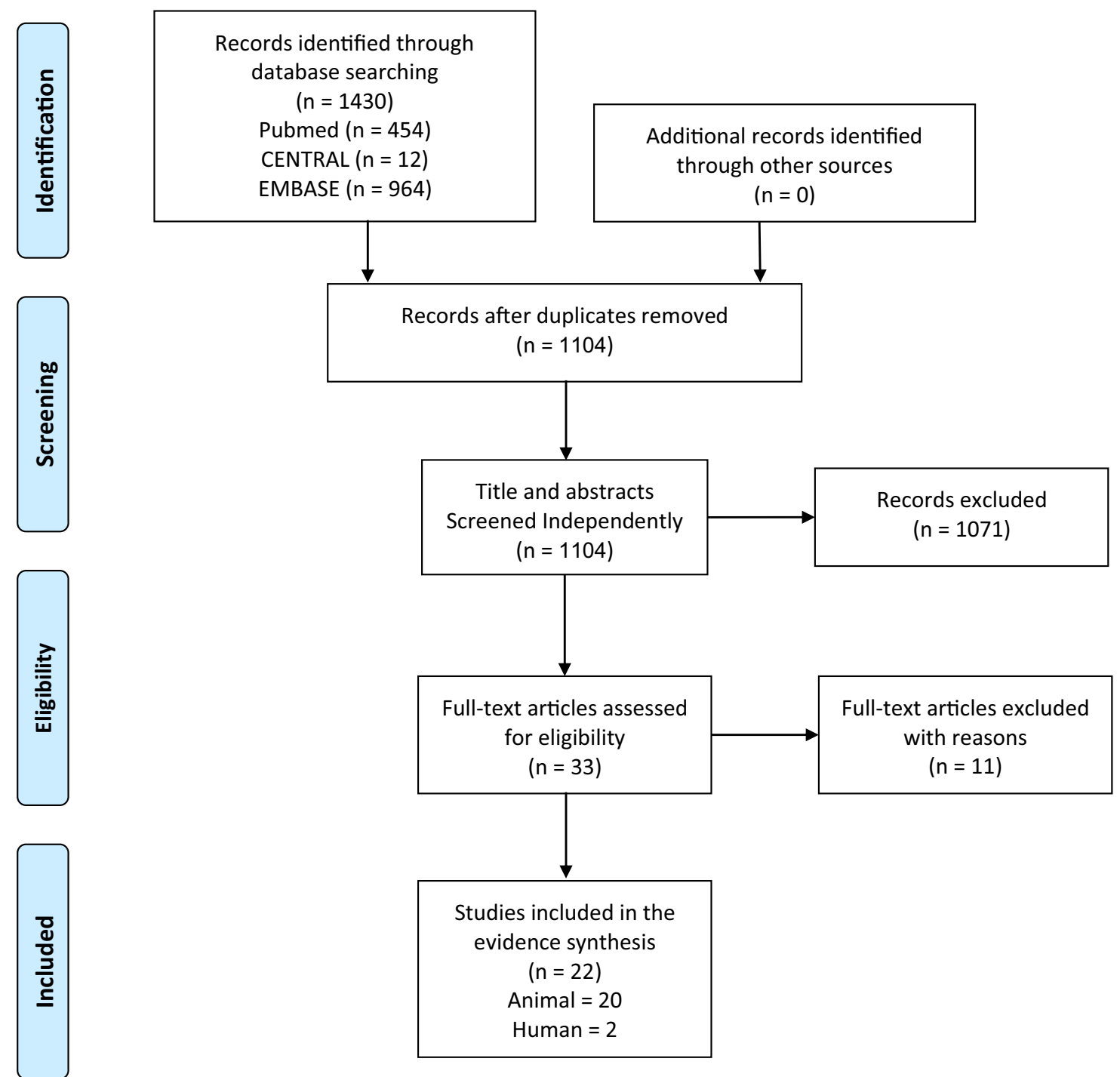

From: Moher D, Liberati A, Tetzlaff J, Altman DG, The PRISMA Group (2009). Preferred Reporting /tems for Systematic Reviews and MetaAnalyses: The PRISMA Statement. PLoS Med 6(7): e1000097. doi:10.1371/journal.pmed1000097

For more information, visit www.prisma-statement.org.

Fig. 1 PRISMA flow diagram 


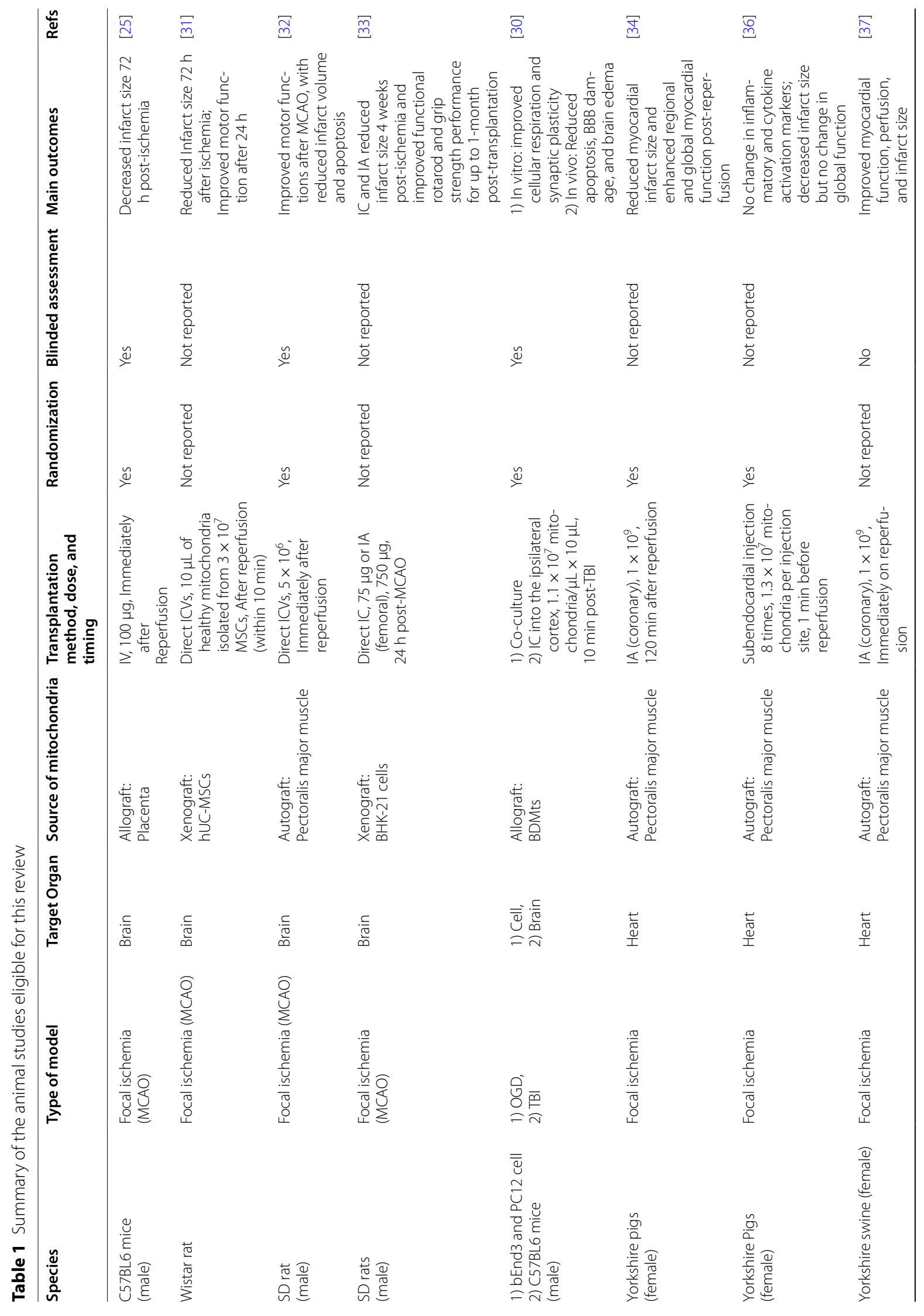




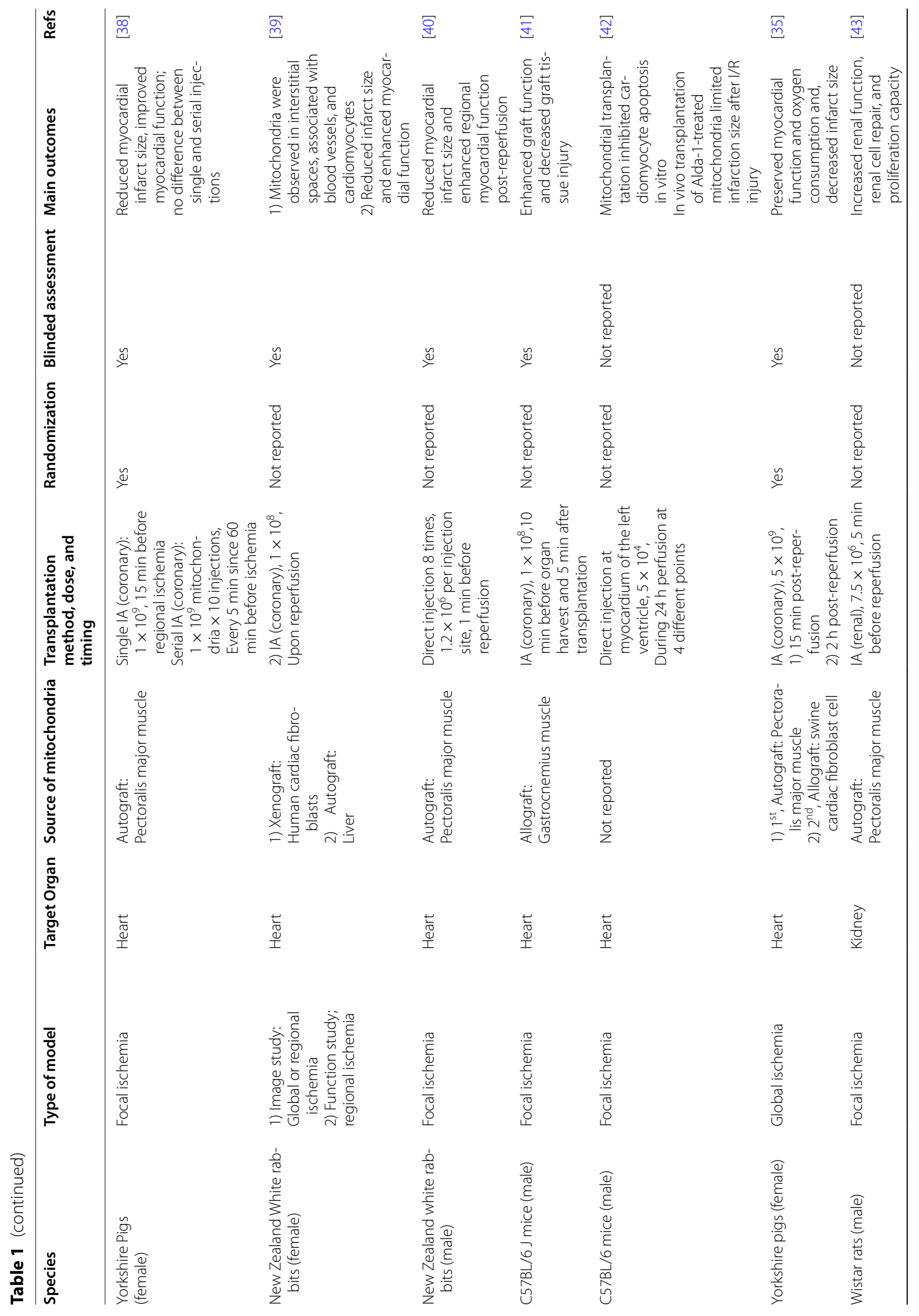




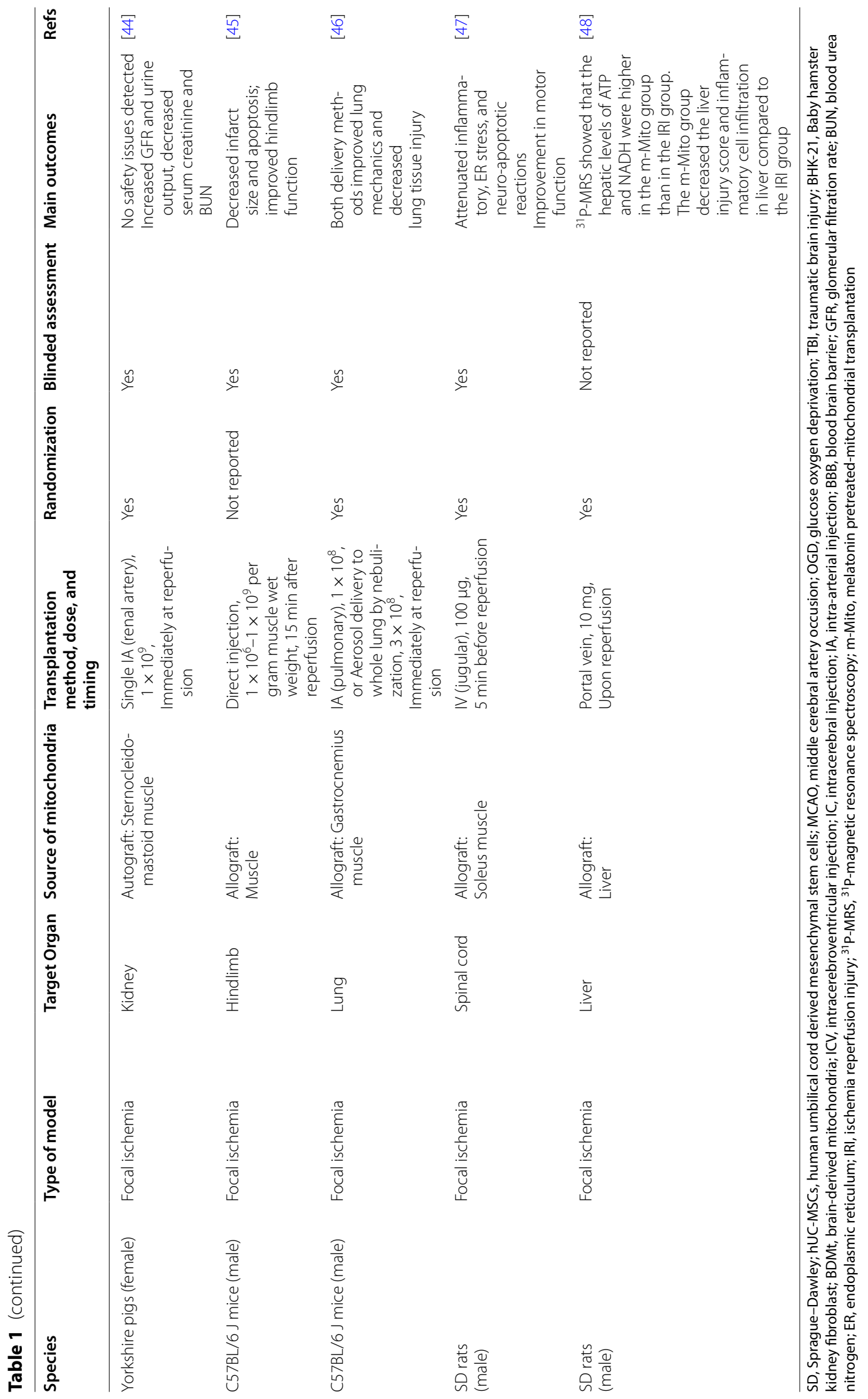


One animal study demonstrated the benefits of mitochondrial transplantation in the setting of TBI. TBI is known to result in inflammatory and oxidative stress as well as mitochondrial dysfunction that occurs shortly after the initial impact. Using both a cellular glucoseoxygen deprivation model in bEnd3 and PC12 cells and an in vivo mouse model of TBI using a controlled cortical impact device, Zhang et al. [30] suggested that administration of exogenous brain-derived mitochondria improved cellular respiration and the expression of proteins associated with synaptic plasticity in vitro, along with increased angiogenesis; reduction in apoptosis, brain edema, and blood brain barrier leakage; and improved long-term potentiation in the hippocampus, at 7 days after in vivo TBI.

All the studies showed improvement in the primary outcome with mitochondrial transplantation. All the above-mentioned studies demonstrated an improvement in brain function following mitochondrial transplantation. Of these, two out of five studies did not report whether the study was randomized or if the study was conducted in a blinded manner; the remaining studies included information regarding both randomization and blinding. The sources of donor organs, types of transplantation, timing of transplantation, and transplant methods varied across all studies.

\section{Cardiac IRI model}

Nine experimental models of cardiac IRI were undertaken in pigs [34-38], rabbits [39, 40], and mice [41, 42], including seven focal ischemia models [34, 36-42] and one global ischemia model [35]. The types of transplantation were autologous [34-40] and allograft [41]. Administration was intraarterial $[34,35,37-39,41]$ and through direct injection $[36,40]$.

We reviewed seven animal studies that demonstrated the benefits of mitochondrial transplantation in focal cardiac ischemia models. Blitzer et al. [34] used Yorkshire pigs that underwent $30 \mathrm{~min}$ of ischemia, which was induced by snaring the left anterior descending artery, to test the efficacy of delayed mitochondrial transplantation. After the initial $120 \mathrm{~min}$ of reperfusion, swine were randomly assigned to receive either autologous mitochondria $\left(1 \times 10^{9}\right.$ in $5 \mathrm{~mL}$ of vehicle) or vehicle only $(5 \mathrm{~mL})$ delivered antegrade as a bolus to the left coronary ostium. Echocardiographic analysis demonstrated that the hearts of pigs receiving delayed mitochondrial transplantation after IRI showed enhanced ejection fraction, fractional shortening, and fractional area change at $240 \mathrm{~min}$ of reperfusion compared with those in the untreated pigs. Although no significant differences were found between the groups in the area at risk, the infarct size was significantly decreased in the hearts of treated pigs [34]. Kaza et al. [36] injected autologous mitochondria into the area at risk (AAR) of the heart after 24 min of temporary regional ischemia, which was induced by snaring the circumflex artery in a pig model. The mitochondria isolated from the dissected pectoralis major muscle were injected into the AAR $\left(1.3 \times 10^{7}\right.$ per injection site $\times 8$ injection site), which significantly enhanced myocardial cell viability and reduced the infarct size. Magnetic resonance imaging showed that the injected mitochondria were present for at least four weeks after the injection [36]. Shin et al. [37] demonstrated that autologous mitochondria $\left(1 \times 10^{9}\right.$ mitochondria) injected into the left coronal artery after $30 \mathrm{~min}$ of regional ischemia, which was induced by temporary snaring of the mid left anterior descending artery, significantly improved myocardial function, coronary blood flow, and infarct size. The authors thus concluded that intracoronary delivery of mitochondria is a safe and efficacious therapy for myocardial ischemia-reperfusion injury [37]. Guariento et al. [38] performed autologous mitochondrial transplantation as a therapeutic strategy for prophylactic myocardial protection in a porcine model of regional IRI. Yorkshire pigs received vehicle or mitochondria either as a single bolus $\left(1 \times 10^{9}: \mathrm{MT}_{\mathrm{S}}\right)$ or serially (10 injections of $1 \times 10^{9}$ at $5 \mathrm{~min}$ intervals over 60 min: $\mathrm{MT}_{\mathrm{SS}}$ ) prior to cardiac IRI. $\mathrm{MT}_{\mathrm{S}}$ and $\mathrm{MT}_{\mathrm{SS}}$ significantly enhanced coronary blood flow and ejection fraction and significantly reduced the infarct size. However, no significant difference in AAR was observed between the treatment groups. The authors suggest that preischemic mitochondrial transplantation, either by single or serial administration, facilitates myocardial protection from IRI by significantly reducing the infarct size and enhancing global and regional function [38]. Cowan et al. [39] used New Zealand White rabbits to investigate whether exogenous mitochondria can be effectively delivered through the coronary vasculature to protect the ischemic myocardium, and studied the fate of these transplanted organelles in the heart. Xenografted mitochondria were observed in the interstitial spaces and were associated with the blood vessels and cardiomyocytes. They also found that autologous liverderived mitochondria markedly reduced the infarct size and enhanced myocardial function [39]. Masuzawa et al. [40] transplanted mitochondria $\left(9.7 \pm 1.7 \times 10^{6} /\right.$ $\mathrm{mL}$ ), which were autologously derived from the pectoralis major muscle, in rabbits subjected to regional ischemia, $1 \mathrm{~min}$ prior to reperfusion. Regional ischemia was achieved by temporarily narrowing the left anterior descending artery for $30 \mathrm{~min}$. The animals were then allowed to recover for 4 weeks to measure their heart function. Mitochondrial transplantation significantly 
decreased the infarct size, creatine kinase MB levels, cardiac troponin-I levels, and apoptosis in the RI zone compared with the vehicle treatment group. The authors also showed that in vivo and in vitro transplanted mitochondria were observed in the interstitial spaces and were internalized by cardiomyocytes at $2-8$ $\mathrm{h}$ after transplantation. The transplanted mitochondria enhanced oxygen consumption, high-energy phosphate synthesis, and the induction of cytokine mediators and proteomic pathways that are important in preserving myocardial energetics, cell viability, and enhanced postinfarct cardiac function [40]. In a murine heart transplantation model, Moskowitzova et al. [41] reported that $1 \times 10^{8}$ allogeneic mitochondria isolated from the gastrocnemius muscle were delivered antegrade to the coronary arteries via injection to the coronary ostium before harvesting the donor heart and after transplantation. Mitochondrial therapy $\left(1 \times 10^{8}\right.$ in respiratory buffer) prolonged the cold ischemia time, significantly enhanced graft function, and decreased graft tissue injury [41].

We found one study that investigated the potential therapeutic effects of pretreating donor mitochondria for mitochondrial transplantation in myocardial IRI. Aldehyde dehydrogenase 2 (ALDH2) plays a key role in regulating mitochondrial homeostasis. Sun et al. [42] investigated the potential effects of Alda-1, an ALDH2 activator, on mitochondrial transplantation in myocardial IRI. In vitro, mitochondrial transplantation inhibited the cardiomyocyte apoptosis induced by hypoxia-reoxygenation exposure, independent of Alda-1 treatment. In vivo transplantation of Alda-1-treated mitochondria limited the IRI as assessed by echocardiography, 2,3,5-triphenyltetrazolium chloride (TTC) staining, and imaging studies.

One animal study demonstrated the benefits of mitochondrial transplantation in a global cardiac ischemia reperfusion model. In a study by Guariento et al. [35] in 2020, Yorkshire pigs received vehicle or autologous mitochondria as a single bolus $\left(5 \times 10^{9} \mathrm{MT}\right)$, after $15 \mathrm{~min}$ of reperfusion. Another group of animals (serial injection of mitochondria $\left[\mathrm{MT}_{\mathrm{S}}\right]$ ) received a second injection of mitochondria $\left(5 \times 10^{9}\right)$ after $2 \mathrm{~h}$ of ex situ heart perfusion, and was reperfused for an additional $2 \mathrm{~h}$. Both the $\mathrm{MT}$ and $\mathrm{MT}_{\mathrm{S}}$ groups showed significantly increased left ventricle function (ventricular peak developed pressure, maximal ventricular pressure rise, fractional shortening), increased myocardial oxygen consumption, and decreased infarct size at $4 \mathrm{~h}$ post-reperfusion compared with the vehicle-treated group [35].

We did not find any studies that failed to demonstrate an improvement in cardiac function after mitochondrial transplantation. Seven of nine studies did not report either randomization or blinded assessment. In most studies, mitochondria that were autologously derived from the pectoralis major muscle were injected via the coronary artery.

\section{Kidney IRI model}

There were two studies on experimental models of kidney focal ischemia in rats [43] and pigs [44], in which autologous mitochondria were injected intra-arterially.

Jabbari et al. [43] demonstrated that mitochondrial autografts from healthy muscle cells to injured kidney cells through injection into the renal artery prevented renal tubular cell death, restored renal function, ameliorated kidney damage, improved the regenerative potential of renal tubules, and decreased IRI-induced apoptosis [43]. Doulamis et al. [44] investigated the safety and efficacy of autologous mitochondrial transplantation by intra-arterial injection for renal protection in a swine model of bilateral renal IRI. They found that the injected mitochondria were rapidly taken up by the kidneys. After $24 \mathrm{~h}$ of reperfusion, the estimated glomerular filtration rate and urine output were significantly increased, whereas the serum creatinine and blood urea nitrogen levels were significantly decreased by mitochondrial transplantation, along with improved acute histopathological changes. The authors thus suggested that their results provide a basis for clinical translation and will enhance the armamentarium of clinicians for the treatment of acute kidney injury [44].

\section{Other IRI models}

Studies on mitochondrial transplantation have also been conducted in other experimental models including acute limb ischemia [45], lung IRI [46], spinal cord injury [47], and acute liver injury [48] models. Orfany et al. [45] used a C57BL/6 J male mouse acute limb ischemia model to compare the impact of dose-dependent mitochondrial injection $\left(1 \times 10^{6}, 1 \times 10^{7}, 1 \times 10^{8}\right.$, and $1 \times 10^{9}$ mitochondria/g muscle) after IRI with f-Rhodamine 6G-labeled mitochondria. Positron emission tomography imaging demonstrated diffuse mitochondrial uptake into the injected muscle group and muscle histology showed significantly improved infarct size; further, dosedependent significant differences were achieved between the lowest and highest dosing regimens. Mitochondrial injections were associated with improved functional status by visual gait scoring and increased IL-10 expression by multiplex analysis [45]. Moskowitzova et al. [46] investigated the efficacy of mitochondrial transplantation in a murine lung IRI model. Male C57BL/6 J mice received either vehicle or vehicle-containing mitochondria either by vascular delivery through the pulmonary artery or by aerosol delivery via the trachea (nebulization). At 
$24 \mathrm{~h}$ after reperfusion, lung function was found to be increased, whereas tissue injuries were significantly decreased in the mitochondria-treated groups compared with the respective vehicle groups. No significant differences in cytokine and chemokine levels were observed between the groups. The authors thus concluded that mitochondrial transplantation by vascular delivery or nebulization improved lung mechanics and decreased lung tissue injury [46]. Fang et al. [47] tested the neuronal protective effect of transplanting viable mitochondria into the ischemic spinal cord in rats. Mitochondria (100 $\mu \mathrm{g})$ were isolated from the soleus muscle and delivered through the jugular vein before reperfusion. They found that mitochondrial transplantation significantly reduced neuronal apoptosis and improved locomotor function in rats with spinal cord ischemia by suppressing oxidative stress and endoplasmic reticulum (ER) stress [47]. Ko et al. [48] investigated whether ${ }^{31} \mathrm{P}$-magnetic resonance spectroscopy $\left({ }^{31} \mathrm{P}-\mathrm{MRS}\right)$ could accurately identify the protective effects of transplanting melatonin-pretreated mitochondria (m-Mito) on acute liver IRI in rats. At 72 $\mathrm{h}$ after acute liver IRI, ${ }^{31} \mathrm{P}$-MRS showed that the hepatic levels of ATP and NADH were significantly higher in the m-Mito group than in the IRI group. Histopathology revealed that the liver injury score and inflammatory cell infiltration in the liver parenchyma were significantly decreased with the m-Mito-transplanted IRI group [48].

All studies demonstrated improvement in IRI following mitochondrial transplantation. In most studies, mitochondria derived from skeletal muscles were used for allograft transplantation. The transplantation method and timing differed depending on the experimental model used.

\section{Human studies}

In this systematic review, we did not find any randomized controlled trials of mitochondrial transplantation in humans. We identified two human studies that used mitochondrial transplantation, and these were reported by the same study group. The group first published a single-arm interventional case series with autologous mitochondrial transplantation in five pediatric patients who required central extracorporeal membrane oxygenation (ECMO) support, and showed that mitochondrial transplantation is feasible and safe [49]. Thereafter, in 2020, the same group reported their further experience with injection of autologous mitochondria into the myocardium of pediatric patients in a single-center retrospective study [50].

At Boston Children's Hospital (BCH) from May 2002 to December 2018, 10 pediatric patients with severe heart disease who required central ECMO support for IRIassociated myocardial dysfunction after cardiac surgery were eligible for autologous mitochondrial transplantation. These 10 subjects were compared with 14 historical controls.

Mitochondria were harvested from non-ischemic rectus abdominis muscle, and the isolation was performed within 20-30 min under sterile conditions in the cardiac intensive care unit or operating room. A $6 \mathrm{~mm} \times 6 \mathrm{~mm}$ piece of heathy rectus abdominis muscle was harvested and mitochondria were isolated, yielding approximately $2 \times 10^{10}$ viable and respiration competent mitochondria from $0.18 \pm 0.04 \mathrm{~g}$ (wet weight) of tissue sample. The isolated mitochondria were suspended in $1 \mathrm{~mL}$ of respiration buffer at a concentration of approximately $1 \times 10^{8}$ to $1 \times 10^{9}$ particles $/ \mathrm{mL}$. During the same intervention, mitochondria were delivered by direct injection using a tuberculin syringe (28-gauge needle) to the myocardium affected by IRI, as identified by epicardial echocardiography. After the procedure, cardiac function assessed by speckle tracking echocardiography was reviewed by two blinded investigators for median circumferential strain and qualitative assessment.

No patients had adverse short-term complications related to mitochondrial injection (arrhythmia, intramyocardial hematoma, or scarring). Blood parameters (white blood count, serum lactate, creatinine, and blood urea nitrogen) did not differ between patients who underwent mitochondrial transplantation and those who did not receive mitochondrial transplantation. Patients subjected to mitochondrial transplantation were more likely to successfully wean from ECMO and exhibited better ventricular strain magnitude at the time of initial decannulation $(23.0 \%$ vs. $16.8 \%, \mathrm{P}=0.03)$ and lower overall occurrence of cardiovascular events (20\% vs. $79 \%$, $\mathrm{P}<0.01)$ compared to the control group. Kaplan-Meier curves and Cox regression analysis models showed a higher composite estimated risk of cardiovascular events in the control group (hazard ratio, 4.6; 95\% confidence interval, 1.0-20.9; $\mathrm{P}<0.04)$ [50].

\section{Discussion}

\section{Summary of main results}

We identified 20 animal and 2 human studies to provide current evidence for mitochondrial transplantation as a treatment for IRI. In the animal studies, 14 of $20(70.0 \%)$ studies focused on IRI models of either the brain or heart, and $12(60.0 \%)$ studies were performed in a blinded manner. Both autograft and allograft transplantation were used in 17 (85.0\%) animal studies; the study designs were heterogeneous in terms of route of administration, timing of transplantation, and the dosage used. All animal studies reported that mitochondrial transplantation markedly mitigated IRI in the targeted tissues, but there was variation in the biological biomarkers and pathological 
changes. Of these, two studies demonstrated the beneficial effects of pretreating mitochondria as donors on IRI. Overall, irrespective of the experimental model, none of the studies failed to demonstrate an improvement in functional outcomes after mitochondrial transplantation. Further studies are warranted to elucidate the best methods, the best donors, or the best timing of mitochondrial transplantation for IRI.

In this systematic review, we found only two studies reported by the same group, but did not find any randomized controlled trials regarding the effect of mitochondrial transplantation on IRI. The latest study reported was a single-center, non-randomized, retrospective study with historical controls, which should be noted as the major limitations of the study. Further randomized controlled trials are thus required to demonstrate the impact of mitochondrial transplantation in patients with IRI.

\section{Potential mechanisms underlying the beneficial effects of mitochondrial transplantation on IRI}

The mechanisms responsible for mitochondrial transplantation in IRI are yet to be fully elucidated. Astrocytes play a wide variety of roles in the regulation of neurodevelopment, neurotransmission, blood brain barrier formation, and in protection against oxidative stress and excitotoxicity. Recent studies have shown that astrocytes can release and transfer extracellular mitochondrial particles that enter damaged neurons to support neuroprotection and neurorepair through calcium-dependent CD38 signaling [4, 51] (Fig. 2).

In animal models of brain ischemia, transplanted mitochondria were found to be incorporated into neurons [32], astrocytes [33], and microglial cells [33] of the peri-infarct area, resulting in increased overall ATP content and increased expression of complex IV [32]. Mitochondrial transplantation significantly attenuates cellular oxidative stress, apoptosis [32], and decreased astrogliosis [31,32] and microglial activation [31], and promotes neurogenesis after ischemia [32]. Furthermore, fluorescent imaging revealed that in addition to the ischemic brain, infused labeled mitochondria were found in various organs including the lung, liver, kidney, and heart [25]. Thus, further studies are required to determine how intravenous infusion of mitochondria affects other organs outside the central nervous system after stroke. In models of cardiac IRI, transplanted mitochondria have been shown to act both extra- and intra-cellularly [22]. Injected mitochondria were found to be localized near the site of delivery, whereas vascular perfusion of mitochondria resulted in rapid and extensive dispersal throughout the heart [39]. Transplanted mitochondria increased the total tissue ATP content and ATP synthesis [40] as well as enhanced myocardial cell viability [36]. This increase in high-energy molecules acts to improve cardiac function rapidly. Further, the transplanted mitochondria enhanced the biological pathways that are

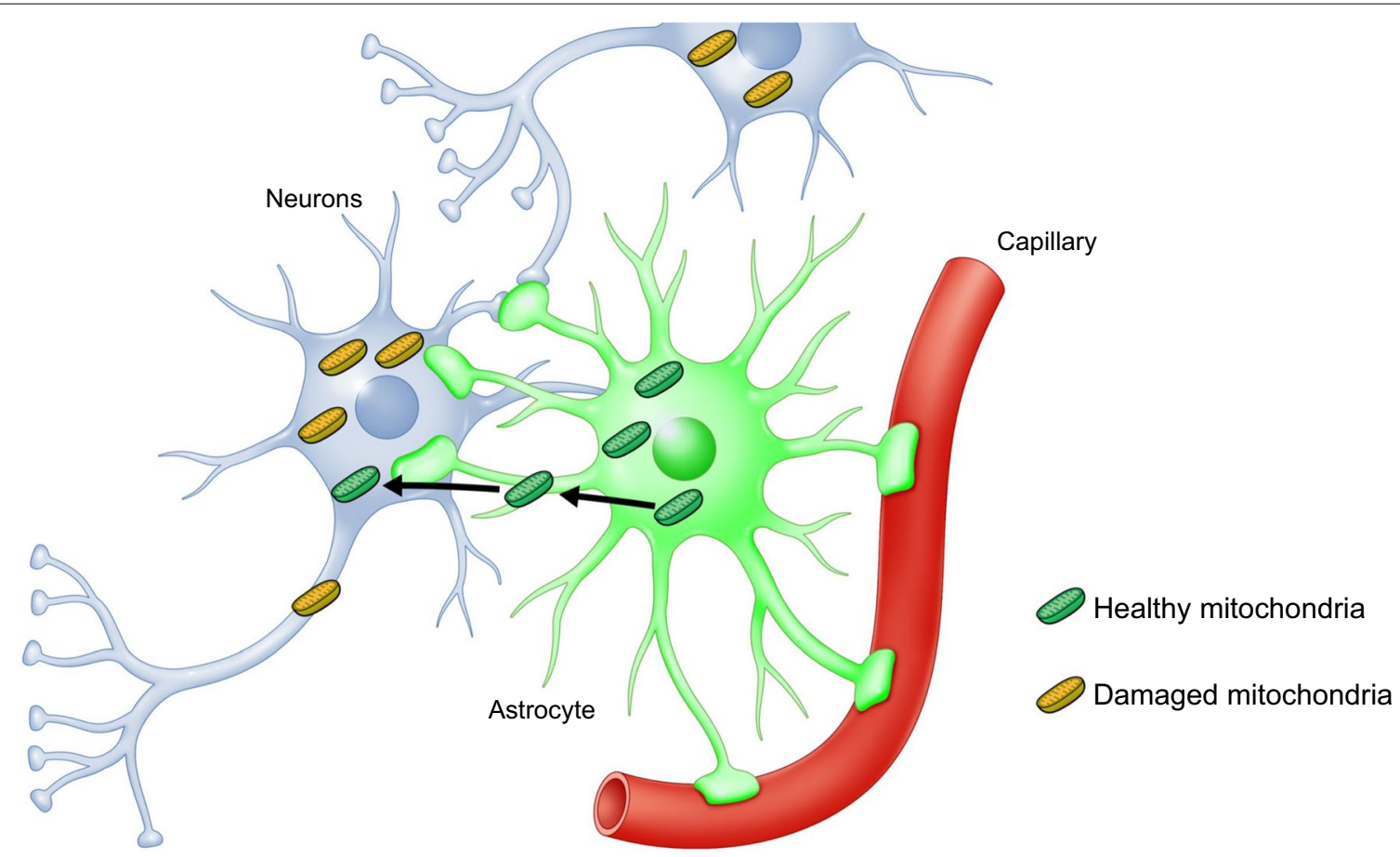

Fig. 2 Mitochondrial transfer from astrocytes to damaged neurons following ischemic insult 
important for preserving myocardial energetics and cell viability [40]. Proteomic analysis has shown upregulation of proteomic pathways for energy production, mitochondrial function, and cellular respiration [40]. Mitochondrial transplantation decreased pro-inflammatory markers [40, 47], increased anti-inflammatory cytokines [45], and inhibited endoplasmic reticulum stress and caspase-3 expression [47]. Mitochondrial transplantation did not alter the levels of pro-inflammatory cytokines $[36,45,46]$. Moreover, metabolomic analysis has indicated that mitochondrial electron transport chain was in the top 10 pathways involved in the altered metabolic profile of the hearts that received mitochondria [35].

The potential mechanisms by which mitochondrial transplantation improves IRI outcomes are shown in Fig. 3. Although the precise mechanisms by which exogenous mitochondria can be taken up by cells remain undetermined, the transplanted exogenous mitochondria are found to successively progress through the endolysosomal system from early endosomes to late endosomes to lysosomes [52]. Most exogenous mitochondria escape from endosomal and lysosomal compartments and effectively fuse with endogenous cardiac cell mitochondria, along with an associated increase in ATP content, oxygen consumption rates, and replacement with depleted mitochondrial DNA [52,
53] (Fig. 3). However, further studies elucidating the molecular mechanism of exogenous mitochondria are warranted to guide the development of this innovative treatment.

From the viewpoint of clinical translation, a potential alternative source for viable, respiratory-competent mitochondria would be from a different individual (allogeneic) or different species (xenogeneic). RamirezBarbieri et al. investigated the immune and damageassociated molecular patterns (DAMPs)-associated response after injections of allogeneic mitochondria in a transplant rejection system of fully MHC-mismatched skin allografts. Their results demonstrated that there is no direct or indirect, acute or chronic alloreactivity, allorecognition or DAMPs reaction to single or serial injections of allogeneic mitochondria [54]. The same group has also shown that in a rabbit model of cardiac IRI the xenogeneic mitochondria transplantation upregulated only cytokines and chemokines which were associated with enhanced post-infarct cardiac function and were not related to alloresponse [40]. All other cytokines and chemokines showed identical levels to baseline at 1, 2, 3 and 4 weeks post mitochondrial injection [40].

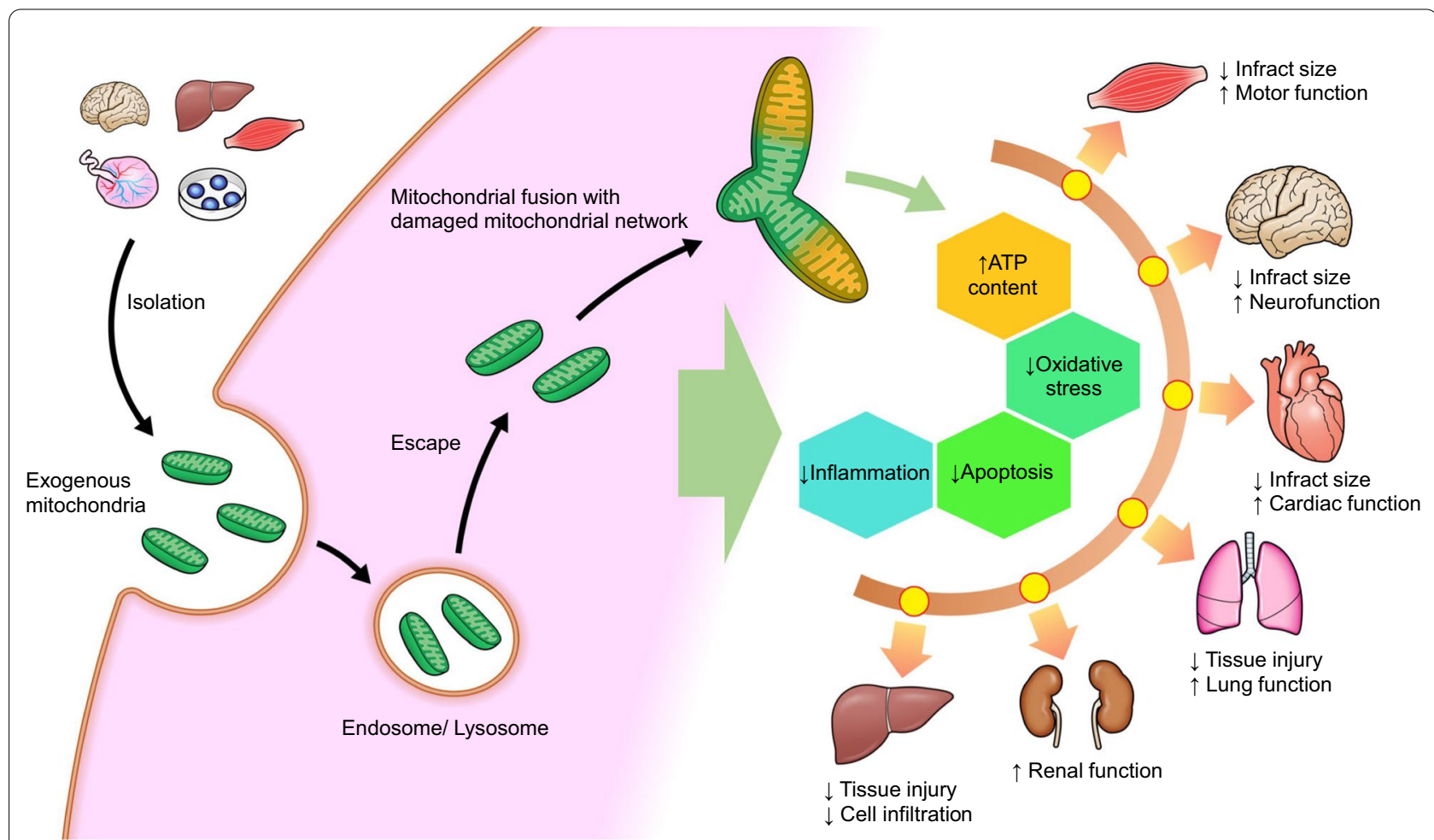

Fig. 3 Potential mechanisms by which mitochondrial transplantation improves outcomes in ischemia-reperfusion injury under critical conditions 


\section{Strength of the review}

A significant strength of this analysis is that, to the best of our knowledge, this study is the first systematic review to provide evidence regarding the potential beneficial effects of mitochondrial transplantation on IRI in critical diseases, in both preclinical and clinical settings. We performed a comprehensive search without any language restrictions. Furthermore, given that recent applications of SR to animal studies revealed poor quality (in terms of randomization and blinding) in most animal studies [26, 55-57], our SR of both preclinical and clinical studies enables scrutiny of the validity of preclinical evidence, helps prevent unnecessary duplication of animal studies, and promotes the clinical translation of this promising concept of mitochondrial transplantation.

\section{Limitations of the review}

The current study has several limitations. First, as the concept of mitochondrial transplantation is in its early stages, we should acknowledge the significant possibility of publication bias, even in animal studies. We also cannot exclude reporting bias in both animal and human studies. This implies that the techniques used may be suboptimal. Second, we did not perform a quantitative meta-analysis because we considered that quantitative meta-analysis was not relevant for the small number of heterogeneous studies included; thus, our data synthesis is mostly descriptive. Third, although we tried to capture a wide range of studies, we may have missed potentially relevant literature. Finally, it should be noted that 12 out of 20 animal studies included in this review used rodent models. It is known that the rodents model has lesser similarities to humans than rabbits and pig models in terms of the physiological, hemodynamical, immune response, and metabolic responses [58, 59].

\section{Conclusion}

In the present review, we have summarized the development and potential applications of mitochondrial transplantation in IRI. The studies included in our analysis have demonstrated the potential of mitochondrial transplantation in the context of IRI in different organs. The provided evidence supports the potential impact of mitochondrial transplantation on IRI in preclinical models, but the relevant clinical translational research remains extremely limited. Therefore, further investigations are required, especially in clinical settings, to explore the mechanism of action and the potential for clinical implementation of mitochondrial transplantation to improve patient outcomes.

\section{Abbreviations}

IRI: Ischemia reperfusion injury; ECMO: Extracorporeal membrane oxygenation; SR: Systematic review; MeSH: Medical subject headings; MCAO: Middle cerebral artery occlusion; TBI: Traumatic brain injury; AAR: Area at risk.

\section{Supplementary Information}

The online version contains supplementary material available at https://doi. org/10.1186/s12967-021-02878-3.

Additional file 1. PRISMA 2009 checklist RT.

Acknowledgements

We would like to thank Prof. Kazuma Yamakawa (Department of Emergency and Critical Care Medicine, Osaka Medical College, Osaka, Japan) for his expert advice regarding this project and systematic review.

\section{Authors' contributions}

Concept and Design: KH. Drafting the manuscript: $\mathrm{KH}$. Acquisition, analysis, and interpretation of data: KH and RT. Data interpretation: MS, CEK, TA, MN, RCC, SJM, and SG. Critical revision of the manuscript: MS, DMR, KJ, KS, EPM, and LBB. All authors read and approved the final manuscript.

Funding

No funding was received for this study.

Availability of data and materials

Not applicable.

\section{Declarations}

Ethics approval and consent to participate

Not applicable.

Consent for publication

Not applicable.

\section{Competing interests}

All authors declare that they have no competing interests.

\section{Author details}

${ }^{1}$ The Feinstein Institutes for Medical Research, Northwell Health System, 350 Community Drive, Manhasset, NY, USA. ${ }^{2}$ Department of Emergency Medicine, North Shore University Hospital, Northwell Health System, 350 Community Dr, Manhasset, NY 11030, USA. ${ }^{3}$ Zucker School of Medicine At Hofstra/Northwell, New York, NY, USA. ${ }^{4}$ Department of Surgery, Northwell Health, Manhasset, NY, USA.

Received: 18 March 2021 Accepted: 7 May 2021

Published online: 17 May 2021

References

1. Liu CS, Chang JC, Kuo SJ, Liu KH, Lin TT, Cheng WL, et al. Delivering healthy mitochondria for the therapy of mitochondrial diseases and beyond. Int J Biochem Cell Biol. 2014;53:141-6.

2. Cipolat S, de Brito O, Dal Zilio B, Scorrano L. OPA1 requires mitofusin 1 to promote mitochondrial fusion. Proc Natl Acad Sci USA. 2004;101(45):15927-32.

3. McCully JD, Cowan DB, Pacak CA, Toumpoulis IK, Dayalan H, Levitsky S. Injection of isolated mitochondria during early reperfusion for cardioprotection. Am J Physiol Heart Circ Physiol. 2009;296(1):H94-105.

4. Hayakawa K, Esposito E, Wang X, Terasaki Y, Liu Y, Xing C, et al. Transfer of mitochondria from astrocytes to neurons after stroke. Nature. 2016;535(7613):551-5. 
5. Shin B, Cowan DB, Emani SM, Del Nido PJ, McCully JD. Mitochondrial transplantation in myocardial ischemia and reperfusion injury. Adv Exp Med Biol. 2017;982:595-619.

6. Murphy MP, Hartley RC. Mitochondria as a therapeutic target for common pathologies. Nat Rev Drug Discov. 2018;17(12):865-86.

7. Nunnari J, Suomalainen A. Mitochondria: In sickness and in health. Cell. 2012;148(6):1145-59.

8. Smith RA, Hartley RC, Cochemé HM, Murphy MP. Mitochondrial pharmacology. Trends Pharmacol Sci. 2012;33(6):341-52.

9. Donnino MW, Liu X, Andersen LW, Rittenberger JC, Abella BS, Gaieski DF, et al. Characterization of mitochondrial injury after cardiac arrest (COMICA). Resuscitation. 2017;113:56-62.

10. Peberdy MA, Andersen LW, Abbate A, Thacker LR, Gaieski D, Abella BS, et al. Inflammatory markers following resuscitation from out-of-hospital cardiac arrest-A prospective multicenter observational study. Resuscitation. 2016;103:117-24

11. Radhakrishnan J, Wang S, Ayoub IM, Kolarova JD, Levine RF, Gazmuri RJ. Circulating levels of cytochrome $\mathrm{c}$ after resuscitation from cardiac arrest: A marker of mitochondrial injury and predictor of survival. Am J Physiol Heart Circ Physiol. 2007;292(2):H767-75.

12. Huang CH, Tsai MS, Hsu CY, Chen HW, Wang TD, Chang WT, et al. Circulating cell-free DNA levels correlate with postresuscitation survival rates in out-of-hospital cardiac arrest patients. Resuscitation. 2012;83(2):213-8.

13. Murphy MP. How mitochondria produce reactive oxygen species. Biochem J. 2009:417(1):1-13

14. Becker LB. New concepts in reactive oxygen species and cardiovascular reperfusion physiology. Cardiovasc Res. 2004;61(3):461-70.

15. Chernorudskiy AL, Zito E. Regulation of calcium homeostasis by ER redox: A close-up of the ER/mitochondria connection. J Mol Biol. 2017;429(5):620-32

16. Reubold TF, Eschenburg S. A molecular view on signal transduction by the apoptosome. Cell Signal. 2012;24(7):1420-5.

17. Wang $X$. The expanding role of mitochondria in apoptosis. Genes Dev. 2001;15(22):2922-33.

18. Callahan LA, Supinski GS. Sepsis induces diaphragm electron transport chain dysfunction and protein depletion. Am J Respir Crit Care Med. 2005;172(7):861-8.

19. Gomes LC, Di Benedetto G, Scorrano L. During autophagy mitochondria elongate, are spared from degradation and sustain cell viability. Nat Cell Biol. 2011;13(5):589-98.

20. Supinski GS, Schroder EA, Callahan LA. Mitochondria and critical illness. Chest. 2020;157(2):310-22.

21. Willmes C. Mitochondria - A powerful therapeutic target. Trends Mol Med. 2020;26(1):1-2.

22. McCully JD, Cowan DB, Emani SM, del Nido PJ. Mitochondrial transplantation: From animal models to clinical use in humans. Mitochondrion. 2017:34:127-34.

23. Hayakawa K, Bruzzese M, Chou SH, Ning M, Ji X, Lo EH. Extracellular mitochondria for therapy and diagnosis in acute central nervous system injury. JAMA Neurol. 2018;75(1):119-22.

24. McCully JD, Levitsky S, Del Nido PJ, Cowan DB. Mitochondrial transplantation for therapeutic use. Clin Transl Med. 2016;5(1):16.

25. Nakamura Y, Lo EH, Hayakawa K. Placental mitochondria therapy for cerebral ischemia-reperfusion injury in mice. Stroke. 2020;51:3142-6.

26. Pound P, Ritskes-Hoitinga M. Can prospective systematic reviews of animal studies improve clinical translation? J Transl Med. 2020;18(1):15.

27. de Vries RB, Wever KE, Avey MT, Stephens ML, Sena ES, Leenaars M. The usefulness of systematic reviews of animal experiments for the design of preclinical and clinical studies. ILAR J. 2014;55(3):427-37.

28. Liberati A, Altman DG, Tetzlaff J, Mulrow C, Gøtzsche PC, loannidis JP, et al The PRISMA statement for reporting systematic reviews and meta-analyses of studies that evaluate health care interventions: Explanation and elaboration. J Clin Epidemiol. 2009;62(10):e1-34.

29. Ouzzani M, Hammady H, Fedorowicz Z, Elmagarmid A. Rayyan-a web and mobile app for systematic reviews. Syst Rev. 2016;5(1):210.

30. Zhang B, Gao Y, Li Q, Sun D, Dong X, Li X, et al. Effects of brain-derived mitochondria on the function of neuron and vascular endothelial cell after traumatic brain injury. World Neurosurg. 2020;138:e1-9.

31. Pourmohammadi-Bejarpasi Z, Roushandeh AM, Saberi A, Rostami MK, Toosi SMR, Jahanian-Najafabadi A, et al. Mesenchymal stem cells-derived mitochondria transplantation mitigates I/R-induced injury, abolishes
1/R-induced apoptosis, and restores motor function in acute ischemia stroke rat model. Brain Res Bull. 2020;165:70-80.

32. Zhang Z, Ma Z, Yan C, Pu K, Wu M, Bai J, et al. Muscle-derived autologous mitochondrial transplantation: A novel strategy for treating cerebral ischemic injury. Behav Brain Res. 2019;356:322-31.

33. Huang PJ, Kuo CC, Lee HC, Shen Cl, Cheng FC, Wu SF, et al. Transferring xenogenic mitochondria provides neural protection against ischemic stress in ischemic rat brains. Cell Transplant. 2016;25(5):913-27.

34. Blitzer D, Guariento A, Doulamis IP, Shin B, Moskowitzova K, Barbieri GR, et al. Delayed transplantation of autologous mitochondria for cardioprotection in a porcine model. Ann Thorac Surg. 2020;109(3):711-9.

35. Guariento A, Doulamis IP, Duignan T, Kido T, Regan WL, Saeed MY, et al. Mitochondrial transplantation for myocardial protection in ex-situperfused hearts donated after circulatory death. J Heart Lung Transplant. 2020;S1053-2498(20):31625-9.

36. Kaza AK, Wamala I, Friehs I, Kuebler JD, Rathod RH, Berra I, et al. Myocardial rescue with autologous mitochondrial transplantation in a porcine model of ischemia/reperfusion. J Thorac Cardiovasc Surg. 2017;153(4):934-43.

37. Shin B, Saeed MY, Esch JJ, Guariento A, Blitzer D, Moskowitzova K, et al. A novel biological strategy for myocardial protection by intracoronary delivery of mitochondria: Safety and efficacy. JACC Basic Transl Sci. 2019;4(8):871-88

38. Guariento A, Blitzer D, Doulamis I, Shin B, Moskowitzova K, Orfany A, et al. Preischemic autologous mitochondrial transplantation by intracoronary injection for myocardial protection. J Thorac Cardiovasc Surg. 2020;160(2):e15-29.

39. Cowan DB, Yao R, Akurathi V, Snay ER, Thedsanamoorthy JK, Zurakowski $D$, et al. Intracoronary delivery of mitochondria to the ischemic heart for cardioprotection. PLOS ONE. 2016;11(8):e0160889.

40. Masuzawa A, Black KM, Pacak CA, Ericsson M, Barnett RJ, Drumm C, et al. Transplantation of autologously derived mitochondria protects the heart from ischemia-reperfusion injury. Am J Physiol Heart Circ Physiol. 2013;304(7):H966-82.

41. Moskowitzova K, Shin B, Liu K, Ramirez-Barbieri G, Guariento A, Blitzer $D$, et al. Mitochondrial transplantation prolongs cold ischemia time in murine heart transplantation. J Heart Lung Transplant. 2019;38(1):92-9.

42. Sun $X$, Gao R, Li W, Zhao Y, Yang H, Chen H, et al. Alda-1 treatment promotes the therapeutic effect of mitochondrial transplantation for myocardial ischemia-reperfusion injury. Bioact Mater. 2021;6(7):2058-69.

43. Jabbari H, Roushandeh AM, Rostami MK, Razavi-Toosi MT, Shokrgozar MA, Jahanian-Najafabadi A, et al. Mitochondrial transplantation ameliorates ischemia/reperfusion-induced kidney injury in rat. Biochim Biophys Acta Mol Basis Dis. 2020;1866:165809.

44. Doulamis IP, Guariento A, Duignan T, Kido T, Orfany A, Saeed MY, et al. Mitochondrial transplantation by intra-arterial injection for acute kidney injury. Am J Physiol Ren Physiol. 2020;319(3):F403-13.

45. Orfany A, Arriola CG, Doulamis IP, Guariento A, Ramirez-Barbieri G, Moskowitzova K, et al. Mitochondrial transplantation ameliorates acute limb ischemia. JVasc Surg. 2020;71(3):1014-26.

46. Moskowitzova K, Orfany A, Liu K, Ramirez-Barbieri G, Thedsanamoorthy JK, Yao R, et al. Mitochondrial transplantation enhances murine lung viability and recovery after ischemia-reperfusion injury. Am J Physiol Lung Cell Mol Physiol. 2020;318(1):L78-88

47. Fang SY, Roan JN, Lee JS, Chiu MH, Lin MW, Liu CC, et al. Transplantation of viable mitochondria attenuates neurologic injury after spinal cord ischemia. J Thorac Cardiovasc Surg. 2021;161(5):e337-47.

48. Ko SF, Chen YL, Sung PH, Chiang JY, Chu YC, Huang CC, Huang CR, Yip HK. Hepatic (31) P-magnetic resonance spectroscopy identified the impact of melatonin-pretreated mitochondria in acute liver ischaemia-reperfusion injury. J Cell Mol Med. 2020;24(17):10088-99.

49. Emani SM, Piekarski BL, Harrild D, Del Nido PJ, McCully JD. Autologous mitochondrial transplantation for dysfunction after ischemia-reperfusion injury. J Thorac Cardiovasc Surg. 2017;154(1):286-9.

50. Guariento A, Piekarski BL, Doulamis IP, Blitzer D, Ferraro AM, Harrild DM, et al. Autologous mitochondrial transplantation for cardiogenic shock in pediatric patients following ischemia-reperfusion injury. J Thorac Cardiovasc Surg. 2020;S0022-5223(20):33142-1.

51. Huang L, Nakamura Y, Lo EH, Hayakawa K. Astrocyte signaling in the neurovascular Unit After Central Nervous System Injury. Int J Mol Sci. 2019;20:2. 
52. Cowan DB, Yao R, Thedsanamoorthy JK, Zurakowski D, Del Nido PJ, McCully JD. Transit and integration of extracellular mitochondria in human heart cells. Sci Rep. 2017;7(1):17450.

53. Pacak CA, Preble JM, Kondo H, Seibel P, Levitsky S, Del Nido PJ, Cowan DB, McCully JD. Actin-dependent mitochondrial internalization in cardiomyocytes: evidence for rescue of mitochondrial function. Biol Open. 2015;4(5):622-6.

54. Ramirez-Barbieri G, Moskowitzova K, Shin B, Blitzer D, Orfany A, Guariento A, Iken K, Friehs I, Zurakowski D, Del Nido PJ, et al. Alloreactivity and allorecognition of syngeneic and allogeneic mitochondria. Mitochondrion. 2019;46:103-15.

55. Hirst JA, Howick J, Aronson JK, Roberts N, Perera R, Koshiaris C, et al. The need for randomization in animal trials: An overview of systematic reviews. PLOS ONE. 2014;9(6):e98856.

56. van der Worp HB, Howells DW, Sena ES, Porritt MJ, Rewell S, O'Collins V, et al. Can animal models of disease reliably inform human studies? PLOS Med. 2010;7(3):e1000245.
57. Henderson VC, Demko N, Hakala A, MacKinnon N, Federico CA, Fergusson $D$, et al. A meta-analysis of threats to valid clinical inference in preclinical research of sunitinib. eLife. 2015;4:e08351.

58. Verma N, Rettenmeier AW, Schmitz-Spanke S. Recent advances in the use of Sus scrofa (pig) as a model system for proteomic studies. Proteomics. 2011;11(4):776-93.

59. Esteves PJ, Abrantes J, Baldauf HM, BenMohamed L, Chen Y, Christensen N, Gonzalez-Gallego J, Giacani L, Hu J, Kaplan G, et al. The wide utility of rabbits as models of human diseases. Exp Mol Med. 2018;50(5):1-10.

\section{Publisher's Note}

Springer Nature remains neutral with regard to jurisdictional claims in published maps and institutional affiliations.
Ready to submit your research? Choose BMC and benefit from:

- fast, convenient online submission

- thorough peer review by experienced researchers in your field

- rapid publication on acceptance

- support for research data, including large and complex data types

- gold Open Access which fosters wider collaboration and increased citations

- maximum visibility for your research: over $100 \mathrm{M}$ website views per year

At BMC, research is always in progress.

Learn more biomedcentral.com/submissions 